\title{
Avaliação Genética de Touros Usando Produção em Lactações Completas ou Parciais Projetadas. 3. Confiabilidade e Ganhos Genéticos ${ }^{1}$
}

\section{Cláudio Manoel Rodrigues de Melo², Tarcisio de Moraes Gonçalves ${ }^{3}$, Mário Luiz Martinez ${ }^{4}$, Rui da Silva Verneque ${ }^{4}$, Antonio Ilson Gomes de Oliveira ${ }^{5}$, Rilke Tadeu Fonseca de Freitas ${ }^{6}$}

RESUMO - Para estudar a viabilidade de se usarem produções em lactações parciais, projetadas, na avaliação do mérito genético de touros, foram utilizadas 4595 lactações de 2254 vacas, filhas de 145 touros de 1618 matrizes, distribuídas em 18 rebanhos, com partos entre 1980 e 1997. A partir de 91, 151, 211 ou 241 dias de lactação, projetaram-se 10, 30, 50 ou 70\% das lactações, para a duração da lactação observada e para 305 dias. As estimativas dos parâmetros genéticos foram obtidas pelo sistema MTDFREML. Incluíram-se no modelo, independente da característica, efeitos fixos de rebanho-ano, época de parto e idade da vaca ao parto, com termos linear e quadrático, considerando-se efeitos aleatórios de animal, efeito permanente de ambiente e erro. A média das confiabilidades, obtida por meio das produções estimadas (PE), variou de 0,60 a 0,67, utilizando-se P305 igual a 0,60. O ganho genético anual pela seleção dos touros utilizando as PE foi, em média, 24,27\% maior que o ganho genético anual da P305, quando as lactações foram projetadas para a duração da lactação observada, e 25,65\% superior, quando as lactações foram projetadas para P305. As confiabilidades obtidas, bem como os ganhos genéticos anuais estimados nas avaliações genéticas, usando as PE, foram semelhantes àquelas obtidas para a produção de leite até 305 dias.

Palavras-chave: avaliação genética, confiabilidade, lactações parciais projetadas, máxima verossimilhança restrita, modelo animal, raça Gir

\section{Sires Genetic Evaluations Using Complete or Partial Projected Lactation Records. 3. Reliabilities and Genetic Gains}

\begin{abstract}
Records of 4595 lactations from 2254 cows, daughters of 145 sires and 1,618 dams of the Gyr breed, assigned to 18 herds, with calving from 1980 to 1997 were used to study the viability of using production from projected partial lactations, on the evaluation. From 91, 151, 211 or 241 days of lactation, 10, 30, 50 or $70 \%$ of the lactations were projected, for the duration of the observed lactation and 305 days of lactation. The estimates of genetic parameters were determined using the MTDFREML system. Independent of the traits, the herd-year, season of calving and age of calving with linear and quadratic parameters were included in the model as fixed effects, and considering the animal, permanent environment and error as random effects. The mean of the obtained reliabilities using the estimation productions (PE) ranged from 0.60 to 0.67 , and the average was obtained using P305 equal to 0.60 . The annual genetic gain for the selection of the sire using the PE was, on the average, 24.27\% higher than the annual genetic gain of P305, when the lactations were projected for the length of the observed lactation, and $25.65 \%$ higher, when the lactations were projected for P305. The recorded reliabilities as well as the annual genetic gain estimated in the genetic evaluations using the PE were similar to those obtained for the production of milk up to 305 days.
\end{abstract}

Key Words: genetic evaluation, Gyr cattle, model animal, partial projected lactation, reliabilities, restricted maximum likelihood

\section{Introdução}

O uso de registros de lactação em progresso na avaliação do mérito genético de touros pode reduzir o tempo requerido para a decisão de quais animais devem ser selecionados.

Um caminho para se analisar a utilidade de registros de produção parcial é determinar a diferença no número de filhas por touro, necessário para se obter a mesma confiabilidade em provas com base em diferentes períodos de lactação em relação às realizadas com informações até 305 dias de lactação. $\mathrm{O}$ principal caminho para esta comparação é a variância dos erros de predição das avaliações de touros (TANDON e HARVEY, 1984).

Segundo TANDON e HARVEY (1984), SETHI e JAIN (1993) e RIBAS e PEREZ (1990), não há muita diferença nas avaliações quanto ao tipo de

\footnotetext{
${ }^{1}$ Parte da Dissertação de Mestrado em Zootecnia.

2 Doutorando em Zootecnia - USP.

3 Professor Assistente da UFLA. e.mail: tarcisio@ufla.br

${ }^{4}$ Pesquisador da Embrapa Gado de Leite.

5 Professor Titular Aposentado da UFLA - Bolsista do CNPq. e.mail: ailson@ufla.br

${ }^{6}$ Professor Adjunto da UFLA. e.mail: rilke@ufla.br
} 
registros de produção utilizados. As diferenças pareceram estar mais dependentes do número de filhas por touro.

Como o intervalo de gerações em bovinos é alto, a confiabilidade da seleção sobre registros parciais, embora possa ser ligeiramente inferior à confiabilidade de avaliações sobre produção de 305 dias, é compensada pela redução no intervalo de gerações, no incremento da intensidade de seleção e no número de filhas por touro nas avaliações genéticas, quando se usam produções de leite de 150 dias de lactação (LAMB e McGILLIARD, 1967; PANDER, HILL e THOMPSON, 1992; e SETHI E JAIN, 1994).

Alta eficiência da seleção usando produções de lactações parciais foi obtida por TANDON e HARVEY (1984), WILMINK (1987) e RIBAS e PEREZ (1990), sendo observada tendência de aumento na mesma com o avanço no estádio da lactação.

Entretanto, pode-se questionar se animais selecionados com base em registros de produções aos 150 dias não terão menores produções, em lactações subseqüentes, em relação àqueles selecionados com base em lactações completas. Segundo VAN VLECK e HENDERSON (1961), existem provas suficientes mostrando que a seleção utilizando lactações parciais não afeta a produção nas lactações posteriores.

O objetivo deste trabalho foi comparar a confiabilidade da predição do valor genético e o ganho genético obtido em avaliações utilizando-se a produção de leite até 305 dias e as produções de lactações projetadas.

\section{Material e Métodos}

A descrição dos dados estudados bem como a metodologia aplicada foram apresentados por MELO et al. (2000).

As avaliações genéticas foram obtidas pelo sistema MTDFREML (BOLDMAN et al., 1995), no qual os dados são analisados sob modelo animal (VAN VLECK, 1992) e usa o método da máxima verossimilhança restrita. Utilizou-se um critério de convergência de $10^{-9}$.

Assumindo intensidade de seleção igual a $1 \mathrm{em}$ todas as produções consideradas, confiabilidade média da avaliação dos 145 touros em cada uma destas produções, desvio-padrão genético aditivo da P305d e intervalo de gerações de 5,$40 ; 5,57 ; 5,74 ; 5,82 ;$ e 6 anos, respectivamente, para as produções estimadas pela projeção de uma porcentagem das lactações a partir de 91, 151, 211 ou 241 dias e para a produção de leite até 305 dias, calculou-se o ganho genético anual $(\Delta G)$ para P305d e PE pela projeção de uma porcentagem das lactações.

Para obter os intervalos de gerações das PE, considerou-se intervalo de gerações de 6 anos para P305d e descontaram-se os dias de projeção das lactações nas PE. Assim, o intervalo de gerações (IG), em anos, foi calculado utilizando a seguinte equação:

$$
\mathrm{IG}=\frac{72-\left(\frac{305-\mathrm{x}_{\mathrm{i}}}{30}\right)}{12}
$$

em que: 72 é o IG em meses de P305d e $x_{i}$, o estádio da lactação a partir do qual as lactações foram projetadas $\left(\mathrm{x}_{\mathrm{i}}=90,150,210\right.$ ou 240 dias $)$.

Para estimar a resposta correlacionada $\left(\mathrm{RC}_{\mathrm{tt}^{\prime}}\right)$ na produção de leite até 305 dias (t), quando a seleção foi realizada por intermédio das $\mathrm{PE}$ pela projeção de uma porcentagem das lactações $\left(\mathrm{t}^{\prime}\right)$, foram usados os valores acima mais as correlações genéticas estimadas entre P305d e as produções estimadas (PE). Foram avaliadas também as eficiências relativas pela seleção indireta (ER).

\section{Resultados e Discussão}

Nas Tabelas 1 e 2, estão apresentadas as médias, os desvios-padrão e as amplitudes das confiabilidades e da variância dos erros de predição das estimativas dos valores genéticos obtidos com as produções de leite estimadas e a P305d.

Nota-se que as médias das confiabilidades das estimativas dos valores genéticos obtidos utilizando as PE pela projeção de porcentagem de lactações, tanto para duração da lactação observada como para 305 dias de lactação, são semelhantes àquela obtida para os valores genéticos obtidos utilizando a P305d. A maior diferença entre as médias de confiabilidade para os valores genéticos obtidos nas avaliações pela produção até 305 dias e as confiabilidades para os valores genéticos obtidos nas avaliações utilizando as produções estimadas foi de 0,07. Estes resultados são similares aos obtidos por TANDON e HARVEY (1984), utilizando registros de primeiras lactações de vacas Holandesas, os quais constataram não haver muita diferença nas avaliações quanto ao tipo de registros de produção utilizados.

Quanto maior a estimativa da variância genética aditiva, menor a fração $\mathrm{PEV} / \hat{\sigma}_{\mathrm{a}}^{2}\left(1+\mathrm{F}_{\mathrm{A}}\right) \mathrm{e}$, conseqüentemente, maior a confiabilidade das estimativas dos valores genéticos, o que é verdadeiro se a variância do erro de predição (PEV) não se alterar ou sua 
722 Rev. bras. zootec.

Tabela 1 - Médias, desvios-padrão (DP) e amplitude das confiabilidades e das variâncias do erro de predição das estimativas dos valores genéticos obtidos utilizando-se das produções estimadas pela projeção de uma porcentagem das lactações para duração da lactação observada e da P305d

Table 1 - Means, standard deviations (SD) and width of the reliabilities and of the variances prediction error of the estimates of the obtained genetic values using the productions estimated by the projection of a percentage for the length of the observed lactation and of milk yield up to 305 days

\begin{tabular}{|c|c|c|c|c|}
\hline \multirow[t]{2}{*}{$\begin{array}{l}\text { Produção estimada } \\
\text { Estimated yield }\end{array}$} & \multicolumn{2}{|c|}{$\begin{array}{c}\text { Confiabilidade } \\
\text { Reliability }\end{array}$} & \multicolumn{2}{|c|}{$\begin{array}{l}\text { Variância do erro de predição } \\
\text { Variance of the prediction error }\end{array}$} \\
\hline & $\begin{array}{c}\text { Média }(\mathrm{DP}) \\
\text { Mean }(D P)\end{array}$ & $\begin{array}{l}\text { Amplitude } \\
\text { Width }\end{array}$ & $\begin{array}{l}\text { Média }(\mathrm{DP}) \\
\text { Mean }(D P)\end{array}$ & $\begin{array}{l}\text { Amplitude } \\
\text { Width }\end{array}$ \\
\hline P305d & $0,60(0,11)$ & $0,42 \mathrm{a} 0,86$ & $204,56(24,31)$ & 133,05 a 236,54 \\
\hline P090d10\%DL & $0,61(0,11)$ & 0,43 a 0,86 & $213,10(25,69)$ & 137,11 a 246,06 \\
\hline P090d30\%DL & $0,64(0,11)$ & 0,45 a 0,88 & $230,12(29,96)$ & 145,26 a 270,64 \\
\hline P090d50\%DL & $0,66(0,10)$ & 0,49 a 0,89 & $250,03(34,59)$ & 154,68 a 297,18 \\
\hline P090d70\%DL & $0,64(0,11)$ & 0,47 a 0,88 & $250,35(32,27)$ & 158,64 a 292,33 \\
\hline P150d $10 \% \mathrm{DL}$ & $0,60(0,11)$ & 0,41 a 0,86 & $205,49(24,14)$ & 134,16 a 237,13 \\
\hline P150d30\%DL & $0,64(0,11)$ & $0,46 \mathrm{a} 0,88$ & $226,98(29,55)$ & 143,29 a 266,78 \\
\hline $\mathrm{P} 150 \mathrm{~d} 50 \% \mathrm{DL}$ & $0,66(0,10)$ & 0,49 a 0,89 & $224,30(33,85)$ & 151,06 a 290,46 \\
\hline P150d70\%DL & $0,64(0,11)$ & 0,47 a 0,88 & $243,17(32,00)$ & 153,00 a 285,36 \\
\hline $\mathrm{P} 210 \mathrm{~d} 10 \% \mathrm{DL}$ & $0,60(0,11)$ & 0,41 a 0,86 & $203,91(23,94)$ & 133,15 a 235,25 \\
\hline $\mathrm{P} 210 \mathrm{~d} 30 \% \mathrm{DL}$ & $0,62(0,11)$ & $0,45 \mathrm{a} 0,87$ & $219,23(27,95)$ & 139,36 a 256,66 \\
\hline $\mathrm{P} 210 \mathrm{~d} 50 \% \mathrm{DL}$ & $0,65(0,11)$ & 0,47 a 0,88 & $229,84(30,65)$ & 143,95 a 271,29 \\
\hline $\mathrm{P} 210 \mathrm{~d} 70 \% \mathrm{DL}$ & $0,64(0,11)$ & 0,46 a 0,88 & $227,88(29,50)$ & 144,13 a 267,10 \\
\hline $\mathrm{P} 240 \mathrm{~d} 50 \% \mathrm{DL}$ & $0,60(0,11)$ & 0,42 a 0,86 & $202,58(24,21)$ & 131,52 a 234,56 \\
\hline $\mathrm{P} 240 \mathrm{~d} 30 \% \mathrm{DL}$ & $0,60(0,11)$ & 0,42 a 0,86 & $197,14(23,28)$ & 128,48 a 227,73 \\
\hline $\mathrm{P} 240 \mathrm{~d} 50 \% \mathrm{DL}$ & $0,60(0,11)$ & 0,42 a 0,86 & $193,96(23,33)$ & 125,66 a 224,83 \\
\hline $\mathrm{P} 240 \mathrm{~d} 70 \% \mathrm{DL}$ & $0,62(0,11)$ & 0,43 a 0,87 & $194,37(24,08)$ & 124,72 a 226,55 \\
\hline
\end{tabular}

Tabela 2 - Médias, desvios-padrão (DP) e amplitude das confiabilidades e das variâncias do erro de predição das estimativas dos valores genéticos obtidos utilizando-se das produções estimadas pela projeção de uma porcentagem das lactações para 305 dias e da produção de leite até 305 dias

Table 2 - Means, standard deviations (SD) and width of the reliabilities and of the variances prediction error of the estimates of the obtained genetic values using the productions estimated by the projection of a percentage of the lactations for 305 days and of milk yield up to 305 days

\begin{tabular}{|c|c|c|c|c|}
\hline \multirow[t]{2}{*}{$\begin{array}{l}\text { Produção estimada } \\
\text { Estimated yield }\end{array}$} & \multicolumn{2}{|c|}{$\begin{array}{l}\text { Confiabilidade } \\
\text { Reliability }\end{array}$} & \multicolumn{2}{|c|}{$\begin{array}{l}\text { Variância do erro de predição } \\
\text { Variance of the prediction error }\end{array}$} \\
\hline & $\begin{array}{l}\text { Média }(\mathrm{DP}) \\
\text { Mean }(D P)\end{array}$ & $\begin{array}{l}\text { Amplitude } \\
\text { Width }\end{array}$ & $\begin{array}{l}\text { Média }(\mathrm{DP}) \\
\text { Mean }(D P)\end{array}$ & $\begin{array}{l}\text { Amplitude } \\
\text { Width }\end{array}$ \\
\hline P090d10\% & $0,61(0,11)$ & 0,43 a 0,87 & $210,73(25,76)$ & 135,82 a 245,04 \\
\hline P090d30\% & $0,63(0,11)$ & 0,45 a 0,88 & $221,96(28,44)$ & 140,86 a 260,22 \\
\hline P090d50\% & $0,65(0,10)$ & 0,48 a 0,89 & $237,21(32,23)$ & 147,64 a 280,89 \\
\hline P090d70\% & $0,64(0,11)$ & 0,47 a 0,88 & $238,00(30,88)$ & 150,51 a 277,98 \\
\hline $\mathrm{P} 150 \mathrm{~d} 10 \%$ & $0,61(0,11)$ & 0,42 a 0,86 & $208,35(25,11)$ & 134,92 a 241,51 \\
\hline P150d30\% & $0,65(0,11)$ & 0,47 a 0,89 & $229,54(30,94)$ & 143,23 a 271,76 \\
\hline $\mathrm{P} 150 \mathrm{~d} 50 \%$ & $0,67(0,10)$ & 0,50 a 0,90 & $244,06(34,66)$ & 149,67 a 291,81 \\
\hline $\mathrm{P} 150 \mathrm{~d} 70 \%$ & $0,67(0,10)$ & 0,50 a 0,89 & $247,25(34,34)$ & 152,83 a 293,14 \\
\hline $\mathrm{P} 210 \mathrm{~d} 10 \%$ & $0,61(0,11)$ & 0,43 a 0,86 & $209,31(25,50)$ & 135,05 a 243,06 \\
\hline $\mathrm{P} 210 \mathrm{~d} 30 \%$ & $0,64(0,11)$ & 0,45 a 0,88 & $222,20(28,86)$ & 140,37 a 261,13 \\
\hline $\mathrm{P} 210 \mathrm{~d} 50 \%$ & $0,65(0,11)$ & 0,47 a 0,89 & $232,88(31,36)$ & 145,37 a 275,45 \\
\hline $\mathrm{P} 210 \mathrm{~d} 70 \%$ & $0,65(0,10)$ & 0,48 a 0,89 & $236,76(32,02)$ & 147,60 a 279,82 \\
\hline P $240 \mathrm{~d} 10 \%$ & $0,60(0,11)$ & 0,42 a 0,86 & $202,68(24,20)$ & 131,64 a 234,59 \\
\hline P240d30\% & $0,60(0,11)$ & 0,41 a 0,86 & $197,14(23,28)$ & 128,48 a 227,74 \\
\hline P240d50\% & $0,60(0,11)$ & 0,42 a 0,86 & $193,48(23,19)$ & 125,50 a 224,16 \\
\hline P240d70\% & $0,61(0,11)$ & 0,43 a 0,87 & $193,07(23,74)$ & 124,21 a 224,76 \\
\hline
\end{tabular}


MELO et al.

alteração for proporcionalmente menor que a da $\hat{\sigma}_{\mathrm{a}}^{2}$, fato verificado neste trabalho (Tabelas 1 e 2). Estes resultados contrariam os obtidos por TANDON e HARVEY (1984), que verificaram pequena perda na precisão das estimativas do mérito genético de touros, quando usaram, para avaliação genética dos touros, registros de produção de 150 dias de lactação, projetados para 305 dias.

Segundo RIBAS e PEREZ (1990) e SETHI e JAIN (1993), é necessário número maior de filhas por touro para se obter a mesma precisão em avaliações, utilizando-se produções de lactações projetadas em relação às avaliações utilizando produções de leite até 305 dias de lactação. Os resultados obtidos no presente trabalho evidenciam ligeira melhoria nas precisões das estimativas com base nas PE em relação àquelas com base em P305d. Este fato, que à primeira vista parece contraditório, pode ser explicado pelas maiores estimativas de variância genética obtidas para as PE em relação à obtida para $\mathrm{P} 305 \mathrm{~d}$.

O ganho genético pode ser usado como critério para comparar o progresso adicional na produção de leite das filhas, pela seleção dos touros com base na produção de leite até 305 dias ou nas produções de lactações parciais projetadas.

Na Tabela 3, estão apresentados o ganho genético anual para a P305d e as PE pela projeção de porcentagem das lactações $(\Delta \mathrm{G})$, a resposta correlacionada na produção de leite até 305 dias, quando a seleção é realizada com base nos valores genéticos previstos com base nas produções estimadas pela projeção de uma porcentagem das lactações $\mathrm{RC}_{\mathrm{tt}^{\prime}}$, e a eficiência relativa pela seleção indireta (ER).

Observa-se que o ganho genético anual pela seleção dos touros utilizando as produções estimadas pela projeção de uma porcentagem das lactações foi, em média, 24,27\% maior que o ganho genético anual da P305d, quando as lactações foram projetadas para a duração da lactação observada, e 25,65\% superior, quando as lactações foram projetadas para 305 dias.

Entretanto, as maiores diferenças estão entre o $\Delta \mathrm{G}$ das lactações projetadas a partir de 91,151 e 211 dias, em que o $\Delta \mathrm{G}$ é maior que o obtido para P305d. A eficiência relativa é uma medida da mudança genética esperada na produção de leite até 305 dias, quando a seleção baseia-se em produções de lactações

Tabela 3 - Ganho genético anual $(\Delta \mathrm{G})$ para produção de leite até 305 dias (P305d) e para as produções estimadas pela projeção de uma porcentagem das lactações, resposta correlacionada no ganho genético da P305d, quando a seleção é realizada com base nas produções estimadas pela projeção de uma porcentagem das lactações $\left(\operatorname{RC}_{t^{\prime}}\right)$, e eficiência relativa pela seleção indireta (ER)

Table 3 - Genetic gain $(\Delta \mathrm{G})$ for milk yield up to 305 days (P305d) and for yields estimated by the projection of a percentage of the lactations, response correlated in the genetic gain of $P 305 d$, when the selection is performed on the basis of yields estimated by the projection of a percentage of the lactations $\left(\mathrm{RC}_{\mathrm{tt}^{\prime}}\right)$ and relative efficiency by the indirect selection (ER)

\begin{tabular}{lccccccc}
\hline $\begin{array}{l}\text { Produção estimada } \\
\text { Estimated yield }\end{array}$ & $\Delta \mathrm{G}$ & $\mathrm{RC}_{\mathrm{t}^{\prime}}$ & $\mathrm{ER}$ & $\begin{array}{c}\text { Produção estimada } \\
\text { Estimated yield }\end{array}$ & $\Delta \mathrm{G}$ & $\mathrm{RC}_{\mathrm{tt}^{\prime}}$ & $\begin{array}{c}\mathrm{ER} \\
E R\end{array}$ \\
\hline P305d & 26,04 & - & - & - & - & - & - \\
P090d10\%DL & 30,71 & 26,47 & 1,02 & P090d10\% & 30,60 & 26,30 & 1,01 \\
P090d30\%DL & 36,01 & 27,65 & 1,06 & P090d30\% & 33,95 & 27,34 & 1,05 \\
P090d50\%DL & 41,60 & 28,55 & 1,10 & P090d50\% & 38,52 & 28,21 & 1,08 \\
P090d70\%DL & 39,18 & 26,20 & 1,01 & P090d70\% & 37,39 & 26,14 & 1,00 \\
P150d10\%DL & 28,05 & 26,04 & 1,00 & P150d10\% & 29,19 & 26,47 & 1,02 \\
P150d30\%DL & 34,45 & 27,72 & 1,06 & P150d30\% & 35,96 & 28,19 & 1,08 \\
P150d50\%DL & 39,44 & 28,61 & 1,10 & P150d50\% & 40,51 & 28,92 & 1,11 \\
P150d70\%DL & 37,21 & 26,89 & 1,03 & P150d70\% & 40,68 & 27,68 & 1,06 \\
P210d10\%DL & 27,01 & 26,03 & 1,00 & P210d10\% & 28,58 & 26,46 & 1,02 \\
P210d30\%DL & 31,00 & 26,88 & 1,03 & P210d30\% & 32,69 & 27,73 & 1,06 \\
P210d50\%DL & 34,78 & 28,18 & 1,08 & P210d50\% & 35,41 & 28,20 & 1,08 \\
P210d70\%DL & 33,54 & 27,39 & 1,05 & P210d70\% & 36,12 & 27,50 & 1,06 \\
P240d10\%DL & 26,63 & 26,04 & 1,00 & P240d10\% & 26,63 & 26,04 & 1,00 \\
P240d30\%DL & 25,81 & 26,04 & 1,00 & P240d30\% & 25,80 & 26,04 & 1,00 \\
P240d50\%DL & 25,56 & 26,01 & 1,00 & P240d50\% & 25,45 & 25,98 & 1,00 \\
P240d70\%DL & 26,76 & 26,91 & 1,03 & P240d70\% & 26,07 & 26,34 & 1,01 \\
\hline
\end{tabular}


projetadas. Tomando a unidade como eficiência relativa padrão, nota-se que a ER para a seleção realizada com base em lactações, projetadas a partir de 91 , 151, 211 ou 241 dias para duração da lactação observada, foi, em média, 4,$75 ; 4,75 ; 4,00 ;$ e $0,75 \%$ superior à padrão, respectivamente. Pela projeção das lactações a partir de $91,151,211$ ou 241 dias para 305 dias, a ER foi, em média, 3,50; 6,75; 5,50; e 0,25\% superior à padrão, respectivamente.

Assim, teoricamente, pode-se inferir que a seleção dos touros com base em produções projetadas proporciona maior $\Delta \mathrm{G}$ na produção de leite até 305 dias que a seleção com base nesta produção. Entretanto, é importante ressaltar que a ER observada para várias produções estimadas, especialmente pela projeção das lactações a partir de 241 dias, é igual à unidade, indicando que a seleção dos touros com base nestas produções eqüivale àquela obtida quando a seleção é praticada com base na produção de leite até 305 dias.

Observa-se, ainda, que a projeção de 50 ou $70 \%$ das lactações, a partir de 91, 151 ou 211 dias, afeta mais o ganho genético que os demais percentuais de projeção.

Existem poucos estudos sobre a eficiência de seleção, quando são usadas lactações parciais como critério de seleção. Entretanto, MADDEN et al. (1995), LAMD e MCGILLIARD (1967), WILMINK (1987) e RIBAS e PEREZ (1990) observaram alta eficiência de seleção por lactações parciais, porém sempre inferior à unidade. Os autores supracitados observaram, ainda, tendência de aumento na eficiência da seleção, com o avanço no estádio da lactação, resultado que contradiz o observado neste estudo, em que a eficiência relativa pela seleção indireta foi sempre superior à unidade, observando-se tendência de redução com o avanço do estádio a partir do qual as lactações foram projetadas. Mais uma vez os resultados obtidos no presente estudo estão relacionados às maiores estimativas de variância genética obtidas para as produções estimadas em relação à obtida para P305d.

\section{Conclusões}

O uso das produções de leite projetadas é extremamente viável nas avaliações genéticas, pois leva a ganhos genéticos anuais, bem como a confiabilidades, similares àqueles obtidos para a produção de leite até 305 dias.

\section{Referências Bibliográficas}

BRASIL. Ministério da Agricultura. Secretaria Nacional de Produção Agropecuária. Portaria número 45 de 10 de outubro 1986. Normas Técnicas para Execução do Serviço de Controle Leiteiro em Bovídeos. Diário Oficial [da República Federativa do Brasil], Brasília, 124(198):15533-15535, 15 de out. 1986. Seção 1.

BOLDMAN, K.G., KRIESE, L.A., VAN VLECK, L.D. et al. 1995. A manual for of MTDFREML: a set of programs to obtain estimates of variances and covariances (DRAFT). Washington USDA, Agricultural Research Service. 115p.

EVERETT, R.W.; CARTER, H.W. 1968. Accuracy of test interval method of calculating dairy herd improvement association records. J. Dairy Sci., 51(12):1936-1941.

GONÇALVES, T.M., MARTINEZ, M.L., MILAGRES, J.C. 1997. Curva de lactação na raça Gir. 2. Influência dos fatores de meio ambiente, estimativas de repetibilidade e herdabilidade para os parâmetros da curva de lactação quadrática logarítmica. R. Bras. Zootec., 26(1):88-97.

LAMB, R.C., McGILLIARD, L.D. 1967. Usefulness of part records to estimate the breeding values of dairy cattle. J. Dairy Sci., 50(9):1458-1467.

MADDEN, D.E., LUSH, J.L., McGILLIARD, L.D. 1955. Relations between parts of lactations and producing ability of Holstein cows. J. Dairy Sci., 38(11):1264-1271,

MELO, C. M.R., OLIVEIRA, A.I.G., MARTINEZ, M.L. et al. 2000. Avaliação genética de touros usando produção em lactações completas ou parciais projetadas. 1. Estimativa de parâmetros genéticos. Rev. bras. zootec., 29(3):707-714

PANDER, B.L., HILL, W.G., THOMPSON, R. 1992. Genetic parameters of test day records of British Holstein - Friesian heifers. Anim. Prod., 55(1):11-21.

RIBAS, M.; PEREZ, B. 1990. Monthly test day milk records and yield at 244 days. II. Genetic parameters in first lactation. Cuban J. Agric. Sci., 24(2):129-144.

SETHI, I.C., JAIN, J.P. 1994. Preliminary selection of sires on partial records in dairy cattle. Biometrical J., 36(3):363-371.

SETHI, I.C., JAIN, J.P. 1993. Sire evaluation on partial records in dairy cattle. Ind. J. Anim. Sci., 63(8):869-872.

STATISTICAL ANALYSIS SYSTEM - SAS. 1995. User's guide: statistics. 5.ed. Cary, 1290p.

TANDON, P.K., HARVEY, W.R. 1984. Best linear unbiased prediction of sire breeding values from part lactations of daughters. J. Dairy Sci., 67(10):2399-2406.

VAN VLECK, L.D. 1992. Animal model for bull and cow evaluation. In: Large dairy herd management symposium. Gainesville. p.1-31.

VAN VLECK, L.D., HENDERSON. C.R. 1961. Use of part lactation records in sire evaluation. J. Dairy Sci., 44(8):15111518.

WILMINK, J.B.M. 1987. Efficiency of selection for different cumulative milk, fat, and protein yields in first lactation. Lvstck. Prod. Sci., (17):211-224.

Recebido em: 01/07/99

Aceito em: 21/10/99 